For $c$ satisfying $0 \leq c \leq 1$ it follows that

$$
\begin{aligned}
\int_{1-c}^{1} x^{n} d x & =\int_{0}^{1} x^{n} d x-\int_{0}^{1-c} x^{n} d x \\
& =A_{n}\left[1-(1-c)^{n+1}\right] \\
& =A_{n}\left[(n+1) c-\frac{n(n+1)}{2} c^{2}+\cdots+(-1)^{n} c^{n+1}\right] .
\end{aligned}
$$

But by reflecting in the line $x=1 / 2$, we also obtain

$$
\begin{aligned}
\int_{1-c}^{1} x^{n} d x & =\int_{0}^{c}(1-x)^{n} d x \\
& =\int_{0}^{c} 1-n x+\frac{n(n-1)}{2} x^{2}-\cdots+(-1)^{n} x^{n} d x \\
& =c A_{0}-n c^{2} A_{1}+\frac{n(n-1)}{2} c^{3} A_{2}-\cdots+(-1)^{n} c^{n+1} A_{n} .
\end{aligned}
$$

Since the two polynomials (1) and (2) in $c$ agree for all $c$ in $[0,1]$, they must be identical. Comparing their linear terms gives the required result $A_{n}=1 /(n+1)$.

ACKNOWLEDGEMENTS. I would like to thank David Tacon for sparking my interest in Cavalieri's arguments, and both Ilan Vardi and the referee for helpful historical remarks.

\title{
REFERENCES
}

1. C. H. Edwards, Jr., The Historical Development of the Calculus, Springer-Verlag, New York, 1979.

2. T. H. Heath, A History of Greek Mathematics, vol. 2, Dover Publications, New York, 1981.

3. T. H. Heath, The Works of Archimedes with the Method of Archimedes, Dover Publications, New York, 1897.

4. D. J. Struik, ed., A Source Book in Mathematics, 1200-1800, Harvard University Press, Cambridge, 1969.

5. I. Vardi, What is ancient mathematics?, Math. Intelligencer 21 (3) (1999) 38-47.

School of Mathematics UNSW Sydney 2052 Australia

n.wildberger@unsw.edu.au

\section{On Euler's Constant—Calculating Sums by Integrals}

\section{Li Yingying}

1. INTRODUCTION. Euler's constant $\gamma$ is defined by

$$
\gamma=\lim _{n \rightarrow \infty} D_{n}
$$


where

$$
D_{n}=\sum_{k=1}^{n} \frac{1}{k}-\log (n+1)
$$

for $n$ in $\mathbb{N}$. Write

$$
r_{n}=\gamma-D_{n}
$$

R. M. Young [1] gave the following estimate for $r_{n}$ :

$$
\frac{1}{2(n+1)}<r_{n}<\frac{1}{2 n}
$$

D. W. DeTemple [2] considered

$$
\tilde{D}_{n}=\sum_{k=1}^{n} \frac{1}{k}-\log \left(n+\frac{1}{2}\right)
$$

in place of $D_{n}$ and showed that

$$
\frac{7}{960} \cdot \frac{1}{(n+1)^{4}}<\gamma-\tilde{D}_{n}+\frac{1}{24\left(n+\frac{1}{2}\right)^{2}}<\frac{7}{960 n^{4}} .
$$

An earlier discussion of $D_{n}$ can be found in Rippon [3]. Furthermore, DeTemple and Wang [4] established an estimate for $r_{n}$ in which Bernoulli numbers are involved.

In this note we use an elementary method to give an exact representation of $r_{n}$, from which asymptotic estimates for $r_{n}$ are then derived. Our method is to calculate sums by means of integrals.

2. THE METHOD. Rewrite $D_{n}$ as

$$
D_{n}=\sum_{k=1}^{n}\left(\frac{1}{k}-\int_{k}^{k+1} \frac{1}{x} d x\right)=\sum_{k=1}^{n} \int_{0}^{1} \frac{t}{k(k+t)} d t .
$$

From (2) we obtain

$$
\begin{aligned}
r_{n} & =\sum_{k=n+1}^{\infty} \int_{0}^{1} \frac{t}{k(k+t)} d t \\
& =\sum_{k=n+1}^{\infty} \int_{0}^{1} t\left(\frac{1}{k(k+t)}-\frac{1}{k(k+1)}\right) d t+\int_{0}^{1} t d t \sum_{k=n+1}^{\infty} \frac{1}{k(k+1)} \\
& =\sum_{k=n+1}^{\infty} \int_{0}^{1} \frac{t(1-t)}{k(k+1)(k+t)} d t+\frac{1}{n+1} \int_{0}^{1} t d t
\end{aligned}
$$

Write

$$
r_{1}(n)=\sum_{k=n+1}^{\infty} \int_{0}^{1} \frac{t(1-t)}{k(k+1)(k+t)} d t, \quad a_{1}=\int_{0}^{1} t d t .
$$


Then

$$
r_{n}=r_{1}(n)+\frac{a_{1}}{n+1} .
$$

Moreover,

$$
\begin{aligned}
r_{1}(n)= & \sum_{k=n+1}^{\infty} \int_{0}^{1} \frac{t(1-t)}{k(k+1)(k+t)} d t \\
= & \sum_{k=n+1}^{\infty} \int_{0}^{1} t(1-t)\left(\frac{1}{k(k+1)(k+t)}-\frac{1}{k(k+1)(k+2)}\right) d t \\
& +\sum_{k=n+1}^{\infty} \int_{0}^{1} \frac{t(1-t)}{k(k+1)(k+2)} d t \\
= & \sum_{k=n+1}^{\infty} \int_{0}^{1} \frac{t(1-t)(2-t)}{k(k+1)(k+2)(k+t)} d t \\
& +\sum_{k=n+1}^{\infty} \frac{1}{2} \int_{0}^{1} t(1-t) d t\left(\frac{1}{k(k+1)}-\frac{1}{(k+1)(k+2)}\right) \\
= & \sum_{k=n+1}^{\infty} \int_{0}^{1} \frac{t(1-t)(2-t)}{k(k+1)(k+2)(k+t)} d t+\frac{1}{2} \int_{0}^{1} t(1-t) d t \frac{1}{(n+1)(n+2)}
\end{aligned}
$$

Let

$$
r_{2}(n)=\sum_{k=n+1}^{\infty} \int_{0}^{1} \frac{t(1-t)(2-t)}{k(k+1)(k+2)(k+t)} d t, \quad a_{2}=\frac{1}{2} \int_{0}^{1} t(1-t) d t .
$$

Then

$$
r_{n}=r_{2}(n)+\frac{a_{1}}{n+1}+\frac{a_{2}}{(n+1)(n+2)} .
$$

For $m$ in $\mathbb{N}$ with $m \geq 2$ we have

$$
\begin{aligned}
r_{m}(n) & =\sum_{k=n+1}^{\infty} \int_{0}^{1} \frac{t(1-t)(2-t) \cdots(m-t)}{k(k+1)(k+2) \cdots(k+m)(k+t)} d t, \\
a_{m} & =\frac{1}{m} \int_{0}^{1} t(1-t) \cdots(m-1-t) d t .
\end{aligned}
$$

By induction we get

$$
r_{n}=\sum_{k=1}^{m} \frac{a_{k}}{(n+1)(n+2) \cdots(n+k)}+r_{m}(n) .
$$

From (3) and (5) we learn that

$$
2 a_{2} \sum_{k=n+1}^{\infty} \frac{1}{k(k+1)^{2}}<r_{1}(n)<2 a_{2} \sum_{k=n+1}^{\infty} \frac{1}{k^{2}(k+1)},
$$


from which we derive (using the fact that $a_{2}=1 / 12$ ) the estimate

$$
\frac{1}{12(n+1)(n+2)}<r_{1}(n)<\frac{1}{12 n(n+1)} \text {. }
$$

Hence we arrive via (4) and (7) at

$$
\frac{1}{2(n+1)}+\frac{1}{12(n+1)(n+2)}<r_{n}<\frac{1}{2(n+1)}+\frac{1}{12 n(n+1)},
$$

which is stronger than (1).

From (6) we obtain (for $m \geq 2$ ):

$$
\begin{aligned}
r_{m}(n) & <a_{m+1} \sum_{k=n+1}^{\infty}\left(\frac{1}{(k-1) k \cdots(k+m-1)}-\frac{1}{k(k+1) \cdots(k+m)}\right) \\
& =\frac{a_{m+1}(n-1) !}{(n+m) !}
\end{aligned}
$$

and

$$
r_{m}(n)>\sum_{k=n+1}^{\infty} \frac{(m+1) a_{m+1}(k-1) !}{(k+1)(k+m) !}>\frac{n(m+1) a_{m+1}}{n+2} \sum_{k=n+1}^{\infty} \frac{(k-2) !}{(k+m) !}
$$

Since

$$
\begin{aligned}
\sum_{k=n+1}^{\infty} \frac{(k-2) !}{(k+m) !}= & \sum_{k=n+1}^{\infty} \frac{1}{(k-1) k \cdots(k+m)} \\
= & \sum_{k=n+1}^{\infty} \frac{1}{(m+1)} \\
& \times\left(\frac{1}{(k-1) k \cdots(k+m-1)}-\frac{1}{k(k+1) \cdots(k+m)}\right) \\
= & \frac{(n-1) !}{(m+1)(n+m) !},
\end{aligned}
$$

we have

$$
r_{m}(n)>\frac{a_{m+1} n !}{(n+2)(n+m) !} .
$$

On the other hand, it is obvious from (6) that for $m \geq 2$

$$
\frac{1}{6 m}(m-2) ! \leq a_{m} \leq \frac{1}{6 m}(m-1) !
$$

We conclude that

$$
\frac{1}{6(n+2) m(m+1)\left(\begin{array}{c}
m+n \\
m
\end{array}\right)}<r_{m}(n)<\frac{1}{6 n(m+1)\left(\begin{array}{c}
m+n \\
m
\end{array}\right)}
$$


for $m \geq 2$, where

$$
\left(\begin{array}{c}
m+n \\
m
\end{array}\right)=\frac{m ! n !}{(m+n) !}
$$

Taking into account (7), we see that (8) is also valid for $m=1$. From (8) we infer

$$
\lim _{m \rightarrow \infty} r_{m}(n)=0
$$

We have thus established the following theorem:

Theorem. Let $D_{n}=\sum_{k=1}^{n} k^{-1}-\log (n+1)$ and let $\gamma=\lim _{n \rightarrow \infty} D_{n}$ be Euler's constant. Then

$$
r_{n}=\gamma-D_{n}=\sum_{k=1}^{\infty} \frac{a_{k}}{(n+1) \cdots(n+k)}
$$

where

$$
a_{1}=\frac{1}{2}, \quad a_{k}=\frac{1}{k} \int_{0}^{1} t(1-t) \cdots(k-1-t) d t \quad(k>1) .
$$

Furthermore,

$$
\frac{1}{6(n+2) m(m+1)\left(\begin{array}{c}
m+n \\
m
\end{array}\right)}<r_{n}-\sum_{k=1}^{m} \frac{a_{k}}{(n+1) \cdots(n+k)}<\frac{1}{6 n(m+1)\left(\begin{array}{c}
m+n \\
m
\end{array}\right)}
$$

The referee kindly produced the following table for the numbers $a_{1}, a_{2}, \ldots, a_{8}$ :

$$
\begin{gathered}
a_{1}=\frac{1}{2}, a_{2}=\frac{1}{12}, a_{3}=\frac{1}{12}, \\
a_{4}=\frac{19}{120}, a_{5}=\frac{9}{20}, a_{6}=\frac{863}{504}, \\
a_{7}=\frac{1375}{168}, a_{8}=\frac{33953}{720} .
\end{gathered}
$$

He also pointed out that $a_{k}$ can be expressed in terms of Stirling numbers of the first kind $s(k, j)$ as

$$
a_{k}=\frac{(-1)^{k+1}}{k} \sum_{j=1}^{k} \frac{s(k, j)}{j+1} \text {. }
$$

Our proof is a completely elementary calculation applying integrals to estimate certain sums. The method can be applied to other cases as well.

ACKNOWLEDGEMENTS. The author is grateful to Professor Wang who encouraged her to write this paper; also, she thanks the referee for valuable suggestions and for correcting typographical mistakes. The project was supported by a grant from the Education Ministry of China. 
1. R. M.Young, Euler's constant, Math. Gazette 75 (1991) 187-190.

2. D. W. DeTemple, A quicker convergence to Euler's constant, Amer. Math. Monthly 100 (1993) 468-470.

3. P. L. Rippon, Convergence with pictures, Amer. Math. Monthly 93 (1986) 476-478.

4. D. W. DeTemple and S. H. Wang, Half integer approximations for the partial sums of the harmonic series, J. Math. Anal. Appl. 160 (1991) 149-156.

\section{Life on the Edge}

\section{Alf van der Poorten}

1. INTRODUCTION. One knows that $\log (1-z)=-\sum_{n=1}^{\infty} z^{n} / n$ for $|z| \leq 1$ and $z \neq 1$. Because $1-e^{i \theta}=-2 i \sin (\theta / 2) \cdot e^{i \theta / 2}$ and $-i=e^{-\pi i / 2}$, we see that

$$
\log \left(1-e^{i \theta}\right)=\log (-i)+\log \left(2 \sin \frac{\theta}{2}\right)+\log e^{i \theta / 2}=\log \left(2 \sin \frac{\theta}{2}\right)-i\left(\frac{\pi}{2}-\theta\right),
$$

and on taking real and imaginary parts of $-\log (1-z)$ with $z=e^{i \theta}=\cos \theta+i \sin \theta$, it follows that

$$
\sum_{n=1}^{\infty} \frac{\cos n \theta}{n}=-\log \left(2 \sin \frac{\theta}{2}\right)
$$

and

$$
\sum_{n=1}^{\infty} \frac{\sin n \theta}{n}=\frac{\pi}{2}-\frac{\theta}{2}
$$

for $0<\theta<2 \pi$.

The relevant rule of thumb is that power series can safely be treated as if they were polynomials of [very] high degree provided one stays well away from the boundary of the disc of convergence. So, guessing that $\log \left(1-e^{i \theta}\right)$ has imaginary part $\sum_{n \geq 1}(\sin n \theta) / n=(\pi-\theta) / 2$ for $0<\theta<2 \pi$ is scary stuff requiring the presence of a qualified mathematician.* Do not try it at home.

In fact, oops! What if $\theta$ creeps down to zero? Surely, all the terms of the series become zero? But its purported sum becomes $\pi / 2$ !

2. EVALUATION OF AN INTEGRAL. Not to worry. Look carefully at the graph of $(\sin x) / x$. It's the sine curve wriggling pathetically as it is squeezed between the hyperbolae $x y=1$ and $x y=-1$.

*MGR: Mathematical Guidance Recommended. Possible use of strong technical language and presence of naked singularities. 